\title{
Assessment of neutrophil gelatinase-associated lipocalin as an early biomarker for canine renal ischemia-reperfusion injury
}

\author{
Jun $\mathrm{Cao}^{1,2} \wedge$, Xiaofeng $\mathrm{Lu}^{2}$, Fei Gao ${ }^{3}$, Xinyu Zhang ${ }^{1}$, Xiaoli Xia ${ }^{1}$, Huaichang Sun ${ }^{1}$ \\ ${ }^{1}$ College of Veterinary Medicine, Jiangsu Co-innovation Center for Prevention and Control of Important Animal Infectious Diseases and Zoonoses, \\ Yangzhou University, Yangzhou, China; ${ }^{2}$ School of Life Sciences, Nantong University, Nantong, China; ${ }^{3}$ Ai-Bi Pet Hospital, Nantong, China \\ Contributions: (I) Conception and design: H Sun, J Cao; (II) Administrative support: X Zhang, X Xia; (III) Provision of study materials or patients: J \\ Cao, X Lu, F Gao, X Zhang; (IV) Collection and assembly of data: J Cao, X Lu, F Gao, X Zhang; (V) Data analysis and interpretation: J Cao, X Lu, \\ F Gao, X Zhang; (VI) Manuscript writing: All authors (VII) Final approval of manuscript: All authors. \\ Correspondence to: Huaichang Sun, PhD. College of Veterinary Medicine, Jiangsu Co-innovation Center for Prevention and Control of Important \\ Animal Infectious Diseases and Zoonoses, Yangzhou University, Yangzhou 225009, China. Email: sunh@yzu.edu.cn.
}

Background: The pathological mechanism of ischemia/reperfusion acute kidney injury (I/R-AKI) differs from other forms of AKI. Neutrophil gelatinase-associated lipocalin (NGAL) is a sensitive biomarker for early diagnosis of AKI, but its utility for diagnosis of canine I/R-AKI remains to be evaluated. The aims of this study were to establish an I/R-AKI model in dogs and to evaluate the diagnostic value of NGAL for canine I/R-AKI.

Methods: We randomly divided 12 beagle dogs into a sham and an I/R group. Artery and vein of the left kidneys of I/R group were cross-clamped for $60 \mathrm{~min}$ followed by reperfusion. The kidney samples were analyzed for histopathological lesions. Serum and urinary samples were analyzed for blood urea nitrogen (BUN), serum creatinine ( $\mathrm{sCr}$ ), serum NGAL (sNGAL), urinary creatinine (uCr), and urinary NGAL (uNGAL). Their detection sensitivities and specificities were compared using a receiver operating characteristics (ROC) method. The expression of NGAL in the renal tissues was analyzed by quantitative RT-PCR (qRT-PCR) and immunohistochemical (IHC) analysis.

Results: After I/R, histopathological analysis showed typical AKI lesions in the dog kidneys of the I/R group, but not in the sham group. Compared to that of the sham group, BUN and sCr of the I/R group rose to significant high levels from $24 \mathrm{~h}$ after I/R. Both uNGAL and sNGAL rose rapidly from $2 \mathrm{~h}$, reached to the peak levels at $12 \mathrm{~h}$, and then receded to the pre-operation levels by $72 \mathrm{~h}$ after I/R. The uNGAL/uCr ratio (uNCR) rose rapidly from $2 \mathrm{~h}$ and remained at variably high levels from 6 to $60 \mathrm{~h}$ after I/R. The ROC analysis showed that detection sensitivities of uNCR, uNGAL, and sNGAL were significantly $(\mathrm{P}<0.0001)$ higher than that of $s \mathrm{Cr}$, without significant difference in specificity. The cut-off values of sNGAL, uNGAL and uNCR were 14,642 pg/mL, 6,773 pg/mL, and 6,701 pg/mg, respectively. Both qRT-PCR and IHC analyses confirmed the dynamic expression of NGAL in the dog kidneys with ischemic acute kidney injury (I-AKI).

Conclusions: There is potential for NGAL to be used as a sensitive biomarker for early diagnosis of canine I-AKI.

Keywords: Ischemic acute kidney injury (I-AKI); canine model; neutrophil gelatinase-associated lipocalin (NGAL); diagnostic biomarkers

Submitted Aug 20, 2020. Accepted for publication Nov 17, 2020.

doi: 10.21037/atm-20-6314

View this article at: http://dx.doi.org/10.21037/atm-20-6314

$\wedge$ ORCID: 0000-0001-8323-5232. 


\section{Introduction}

Acute kidney injury (AKI) is defined as sudden reduction in renal function, resulting in the retention of nitrogenous wastes in plasma. The disease is common in dogs, and is associated with high morbidity and mortality (1). Without effective therapeutic and preventive methods, AKI remains as a critical problem, despite significant progress in understanding the pathophysiological mechanisms $(2,3)$. Renal ischemia/reperfusion (I/R) injury is a leading cause of perioperative AKI, caused by the interruption of renal blood flow (ischemia) and subsequent reperfusion $(1,4)$. Canine models for I/R injury have been used for understanding the dynamics of intraoperative renal ischemia and recovery of renal oxygenation during reperfusion (5). Accurate measurement of renal perfusion in the early period of I/R injury can provide important clinical insight into renal function and mortality in acute renal tubular injury. Although urinalysis, blood urea nitrogen (BUN), serum creatinine $(\mathrm{sCr})$ and ultrasonography can be used to diagnose $\mathrm{I} / \mathrm{R}$ injury $(6,7)$, the low sensitivities of these traditional methods can lead to delay in clinical intervention (1). Therefore, there is a need for more precise and sensitive markers to monitor acute tubular necrosis.

By far, there have been a large number of studies aimed at a variety of potential biomarkers of AKI under different conditions, such as interleukin-18 (IL-18) (8), kidney injury molecule-1 (KIM-1) $(9,10)$, cysteine protease inhibitor (Cystatin C) $(11,12)$, liver type fatty acid binding protein (L-FABP) (13), tissue inhibitor of metalloproteinase-2 (TIMP-2), insulin-like growth factor binding protein-7 (IGFBP-7) (13) and neutrophil gelatinase-associated lipocalin (NGAL). NGAL and IL18 are both secreted molecules, while KIM-1 and L-FABP are localized on cell membrane and in lysosomes of renal tubular cells separately $(9,10)$. Although the four molecules can reflect the status of kidney injury, there are distribution differences among them. The detection of IL-18, KIM1 and L-FABP may be followed by the apoptosis of renal tubular cells, while NGAL can be released soon even if the injury cells are detected $(9,10,13)$. Other molecules like canine serum Cys C, TIMP-2 and IGFBP-7 are also used, for their reporting of GFR or cell cycle arrest in kidney, but the usage for AKI diagnoses are still under discussion $(9,13)$. Among the various early AKI diagnosis mentioned above, NGAL has been used as one of the earliest and most sensitive biomarkers for AKI in mice and humans $(14,15)$. The $25-\mathrm{kDa}$ glycoprotein belongs to the lipocalin superfamily, and is identified as a component of neutrophil granules, which is expressed during inflammatory responses and in the epithelial cells of various tissues (16). Normally, urinary NGAL (uNGAL) is expressed at low concentrations, but its expression is induced to significantly high levels by renal tubular epithelial injury. In addition, uNGAL has been identified as a biomarker for canine AKI when undergoing surgical procedures (17). Serum NGAL (sNGAL) has been identified as an accurate biomarker for human AKI during cardiac surgery (18) . However, the utility of NGAL for early diagnosis of canine ischemic AKI (I-AKI) remains to be evaluated. Therefore, the primary aim of this study was to evaluate the utility of NGAL for early diagnosis of I-AKI using the canine model.

We present the following article in accordance with the ARRIVE reporting checklist (available at http://dx.doi. org/10.21037/atm-20-6314).

\section{Methods}

\section{Animals}

The experimental group included 12 male healthy beagle dogs (3-year-old, 10-12 kg) purchased from Jinfeng Experimental Animal Co., Ltd., Jinan, Shandong, China. The dogs were housed individually in stainless steel cages in a room equipped with an air filtering system, provided with the standard feed $(250 \pm 10 \mathrm{~g} / \mathrm{animal} /$ day $)$, and allowed unrestricted access to water. All animal tests were approved by the Experimental Animal Committee of the Nantong University (20190307-012) and were conducted in accordance with the Guidelines for Experimental Animals of the Ministry of Science and Technology (Beijing, China).

\section{Establishment of I-AKI canine model}

The 12 dogs were randomly divided into a sham group $(n=6)$ and I/R group $(n=6)$. Dogs were anesthetized intramuscularly with sodium pentobarbital $(30 \mathrm{mg} / \mathrm{kg})$ and sodium pentobarbital $(5 \mathrm{mg} / \mathrm{kg})$. After intraperitoneal injection with heparin sodium $(3,000 \mathrm{U})$, the dogs were placed on a homothermic blanket at $37^{\circ} \mathrm{C}$. During the surgical procedure, all dogs were administered intravenously with a balanced electrolyte solution $(10 \mathrm{~mL} / \mathrm{kg} / \mathrm{h})$. Following surgery, pain relief was delivered orally in capsules of tramadol hydrochloride 3 times/d at a dose of $5 \mathrm{mg} / \mathrm{kg}$ for 3 consecutive days (19). Mechanical ventilation was maintained with $40 \%$ oxygen and a tidal volume of 


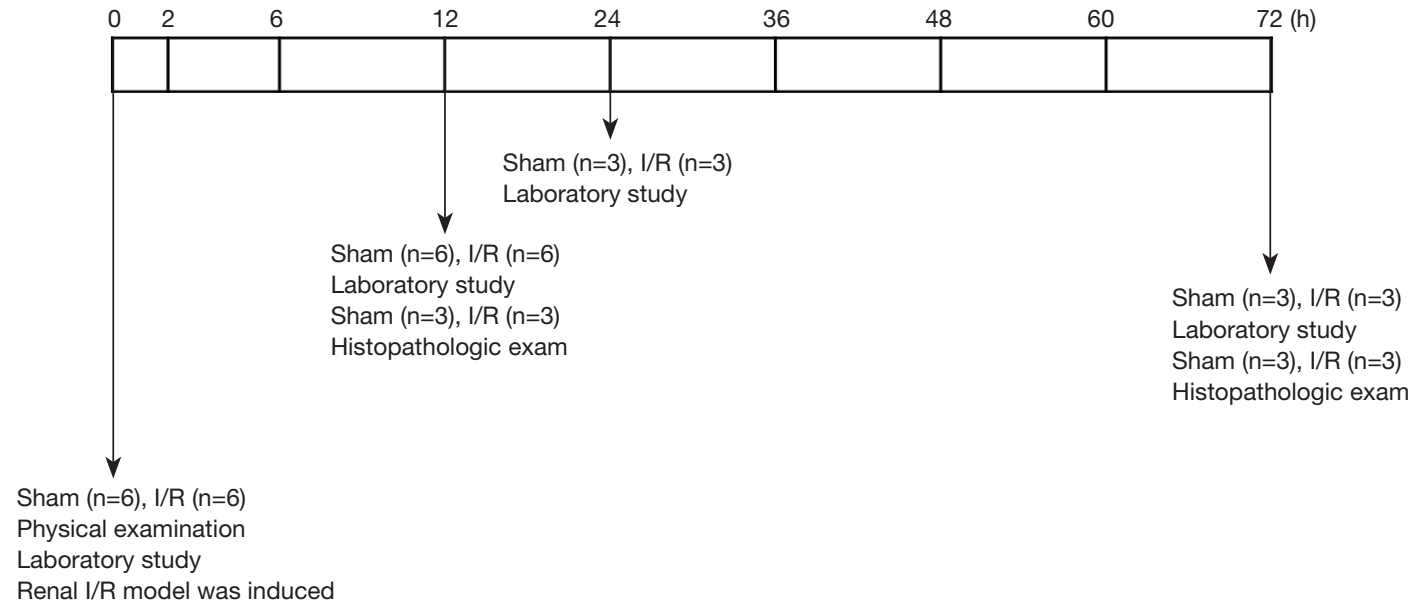

Figure 1 Schedule diagram showing timing of laboratory and histopathologic examinations. Laboratory examinations included blood urea nitrogen (BUN), serum creatinine (sCr), serum neutrophil gelatinase-associated lipocalin (sNGAL), urine NGAL (uNGAL) and uNGAL to creatinine ratio (uNCR), hematoxylin and eosin (H\&E) staining, quantitative polymerase chain reaction (qPCR), and immunohistochemistry (IHC). The number of animals used at each time is shown.

$10 \mathrm{~mL} / \mathrm{kg}$. Expiratory $\mathrm{CO}_{2}$ was maintained at a level of $4.5-5.5 \mathrm{kPa}(20)$.

For dogs in the sham group, the right kidneys were excised by midline laparotomy and transferred immediately into an ice bath as the control samples. For dogs in the I/R group, the right kidneys were excised as the control as described. Both artery and vein of the left kidneys were cross-clamped with an atraumatic vascular clamp, which was removed for reperfusion after $60 \mathrm{~min}(21)$. An $8-\mathrm{Fr}, 55 \mathrm{~cm}$ dog urinary catheter was then inserted, and the incision was closed in 2 layers. After reperfusion, the animals were allowed to wake up and returned to the housing facilities for further recovery. The schedule displayed in Figure 1 shows the timing of laboratory and histopathological examinations.

\section{Sample collection and processing}

Blood and urinary samples were collected at $0,2,6,12$, 24, 36, 48, 60, and $72 \mathrm{~h}$ after surgical operation. The urinary samples were centrifuged for $5 \mathrm{~min}$ at $1,500 \mathrm{~g}, 4^{\circ} \mathrm{C}$, and stored at $-80{ }^{\circ} \mathrm{C}$ before biochemical analysis. The blood samples were centrifuged for $10 \mathrm{~min}$ at 3,000 $\mathrm{g}$ and divided into 2 aliquots, which were analyzed immediately for $\mathrm{sCr}$ and BUN concentrations or stored at $-80{ }^{\circ} \mathrm{C}$ for later biochemical analysis. At 12 and 72 h post I/R, 3 dogs from each group were euthanized with a lethal dose of pentobarbital $(80 \mathrm{mg} / \mathrm{kg})$, and the kidney samples were collected. In brief: blood and urine samples were collected before anesthesia, the renal samples of experimental animals were collected as soon as possible ( $\leq 5 \mathrm{~min}$ ) after anesthesia. Each kidney was sliced into 2 portions and fixed with $10 \%$ formalin for frozen section preparation, or with RNA Keeper-ICE Tissue Transition Buffer (R502-1, Vazyme, Nanjing, Jiangsu, China) for quantitative polymerase chain reaction (qPCR) analysis.

\section{Histopathological analysis}

The frozen sections of formalin-fixed kidney tissues were prepared using Leica CM1950 Cryostat (Leica Microsystems, Wetzlar, Germany) and stained with a hematoxylin and eosin staining kit (Beyotime, Beijing, China) for histological observation (22). Histopathological lesions were scored as previously described; in brief, two sections were taken from each specimen, and 5 fields of view were randomly taken from each section, morphological assessment was first performed by a certified veterinary pathologist who was unaware of the animal treatments and biomarker data. Blind peer review was conducted by another qualified pathologist. The histopathological grade of the lesions was classified as none, minimal, mild, moderate, marked and severe (23).

\section{Biochemical analysis}

Serum Cr and BUN concentrations were measured using 
Table 1 Primer sequences for quantitative RT-PCR analysis in this study

\begin{tabular}{lll}
\hline Gene & Forward primer (5'-3') & Reverse primer (5'-3') \\
\hline NGAL & GGTACGTCATCGGCATAGCA & CCTGAGTAGGGTGGAGGTGA \\
GAPDH & CCCCAATGTATCAGTTGTGGATCTG & CCTGCTTCACTACCTTCTTGATGTC \\
\hline
\end{tabular}

RT-PCR, real-time reverse transcription polymerase chain reaction; NGAL, neutrophil gelatinase-associated lipocalin; GAPDH, glyceraldehyde-3-phosphate dehydrogenase.

VetTest $8008^{\circledR}$ Chemistry Analyzer and VT Analyzer Kit (IDEXX, Lenexa, KS, USA). Urinary creatinine (uCr) was measured using VetTest $8008^{\circledR}$ Chemistry Analyzer and VT Analyzer Kit (IDEXX, Lenexa, KS, USA). Serum NGAL and uNGAL concentrations were measured using NGAL Sandwich ELISA Kit (BioPorto, Hellerup, Denmark), following the manufacturer's instructions.

\section{RT-PCR analysis of NGAL transcription}

Cellular RNA was extracted from the frozen renal tissue using RNA Isolater Total RNA Extraction Reagent (Vazyme, Nanjing, Jiangsu, China) and reverse transcribed using HiScript II Q RT SuperMix (Vazyme, Nanjing, Jaingsu, China) by following the manufacturer's instructions. The quantitative PCR was performed using AceQ qPCR SYBR Green Master Mix (Vazyme, Nanjing, Jiangsu, China) on ABI 7500 Real-Time PCR System with glyceraldehyde-3phosphate dehydrogenase (GAPDH) as the internal control, following the manufacturer's instructions. The primer sequences refer to previous studies (24) and are listed in Table 1.

\section{Immunochemical analysis of NGAL expression}

The frozen sections were incubated with $3 \% \mathrm{H}_{2} \mathrm{O}_{2}$ for $10 \mathrm{~min}$ at room temperature. After washing 3 times with phosphate buffer saline (PBS) ( $\mathrm{pH}$ 7.4), antigen retrieval was performed using the previously described pressure cooker method (25), followed by cooling at room temperature. After blocking with $5 \%$ bovine serum albumin (BSA) in PBS (pH 7.4) for $20 \mathrm{~min}$ at $37^{\circ} \mathrm{C}$, the sections were incubated with monoclonal antibodies (mAb) against canine NGAL (1:50; Vazyme, Nanjing, Jiangsu, China) for $60 \mathrm{~min}$ at $37^{\circ} \mathrm{C}$. After washing again, the sections were stained using Super Vision Immunohistochemical Staining Kit (Boster, Wuhan, Hubei, China) according to the manufacturer's instructions. The images were captured using Leica DM4000B System and analyzed using LASV4.6 Software (Leica Microsystems,
Wetzlar, Germany).

\section{Statistical analysis}

The uNCR was calculated according to formula: uNCR (pg/ $\mathrm{mg})=\mathrm{uNGAL} \times 10^{3} / \mathrm{uCr} \times 112$. The data were tested first by normality test. If the data conformed to a normal distribution, the statistical analysis was performed using the one-way analysis of variance (ANOVA) approach. Otherwise, the statistical analysis was performed using the nonparametric Kruskal-Wallis test with $\mathrm{P}<0.05$ considered to be a significant difference. The urinary and plasma biomarker performance was ascertained using a receiver operating characteristic (ROC) curve analysis (26). Statistical software MedCalc version 19.0.4 (MedCalc Software Ltd., Ostend, Belgium) was used to analyse the ROC curve of the blood and urine concentration of each index to acquire the associated criterion determined by the maximum value of the Youden index, and the sensitivity and specificity of each index are listed (Appendix 1). The calculation formula of the Youden index was:

Youden index $=$ sensitivity + specificity -1

\section{Results}

\section{Histopathological changes}

Dog kidneys from the sham group were histopathologically normal. In contrast, typical AKI was observed in dog kidneys from the I/R group, which were scored as mild or moderate at $12 \mathrm{~h}$, or severe at $72 \mathrm{~h}$ after I/R, the results showed that the scores from the two pathologists were consistent, including tubular cell necrosis, hyaline droplet formation, protein casting, and interstitial edema (Figure 2).

\section{Biochemistry profiles}

Compared to that of the sham dogs, the average BUN concentration of I-AKI dogs was slightly higher before $24 \mathrm{~h}$ and then rose rapidly, reaching the highest level $(20.7 \pm 0.87 \mathrm{mmol} / \mathrm{L}$ ) by $72 \mathrm{~h}$ after I/R (Figure 3$)$. Similarly, 

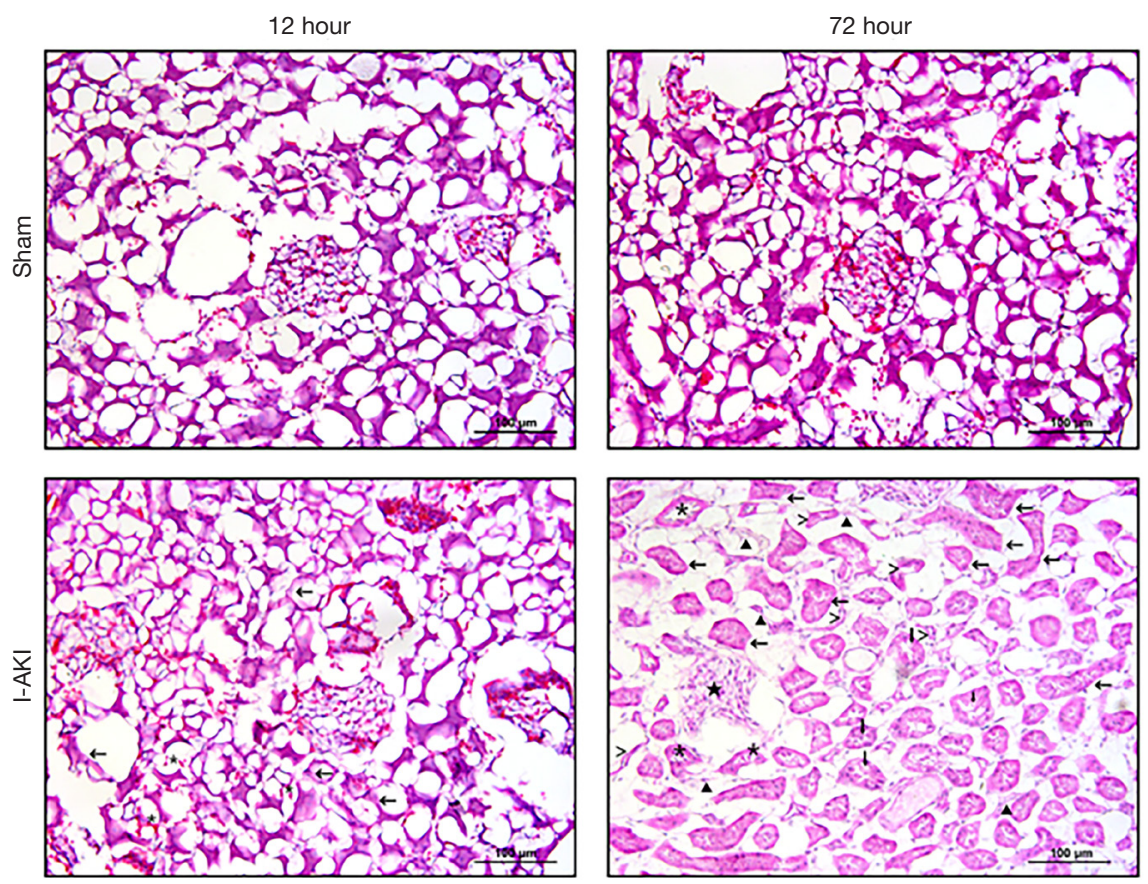

Figure 2 Histopathological changes of dog kidneys with ischemic acute kidney injury (I-AKI). The kidney samples were collected from the sham and ischemia/reperfusion (I/R) groups at 12 and $72 \mathrm{~h}$ after I/R operation, and stained using hematoxylin and eosin (H\&E) method. >, atrophic renal tubules; *, exfoliated cells; $\boldsymbol{\Delta}$, fibrotic tissue; $\downarrow$, protein cast; $\star$, glomerular atrophy; $\leftarrow$, cell swelling, proliferation, lumen stenosis, and brush-like edge disappearance.

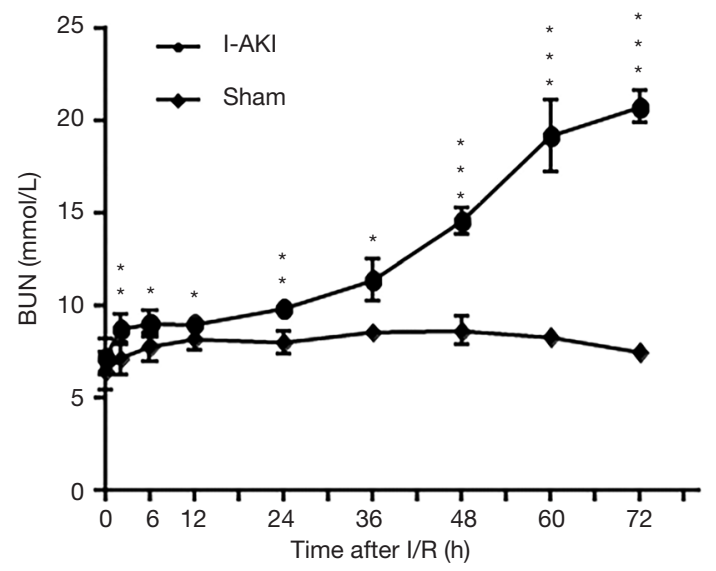

Figure 3 Detection of blood urea nitrogen (BUN) concentrations in sham and ischemic acute kidney injury (I-AKI) dogs. The serum samples were collected at the indicated times after ischemia/ reperfusion (I/R), and BUN was measured using VetTest $8008^{\circledR}$ Chemistry Analyzer and VT Analyzer Kit (IDEXX Laboratories, Lenexa, KS, USA). *, $\mathrm{P}<0.05$; **, $\mathrm{P}<0.01$; ***, $\mathrm{P}<0.001$. the average sCr concentration of I-AKI dogs was slightly higher from $24 \mathrm{~h}$ and then rose rapidly, remaining the highest level $(325.87 \pm 48.63 \mu \mathrm{mol} / \mathrm{L})$ by $60 \mathrm{~h}$ after I/R (Figure 4).

The average uNGAL concentration of the sham dogs was slightly higher at $2 \mathrm{~h}$, and then decreased to the preoperative level from $6 \mathrm{~h}$ after I/R (Figure $5 A$ ). In contrast, the average uNGAL concentration of I-AKI dogs rose quickly as early as $2 \mathrm{~h}$, reached the peak level $(32,742.33 \pm 3,750.90 \mathrm{pg} / \mathrm{mL})$ by $12 \mathrm{~h}$, and then decreased gradually from 24 to $72 \mathrm{~h}$ after I/ $\mathrm{R}$ (Figure $5 A$ ). The average sNGAL concentration of sham dogs was slightly higher from $2-36 \mathrm{~h}$, and then decreased to the preoperative level from $48 \mathrm{~h}$ following I/R (Figure 5B). In contrast, the average sNGAL concentration of I-AKI dogs rose rapidly from as early as $2 \mathrm{~h}$, reached peak level $(63,347.33 \pm 7,163.03 \mathrm{pg} / \mathrm{mL})$ by $12 \mathrm{~h}$, and then decreased gradually from 24 to $72 \mathrm{~h}$ after I/R (Figure $5 B$ ).

The average uNCR value of the sham dogs was slightly higher at $2 \mathrm{~h}$, and then decreased to the preoperative 


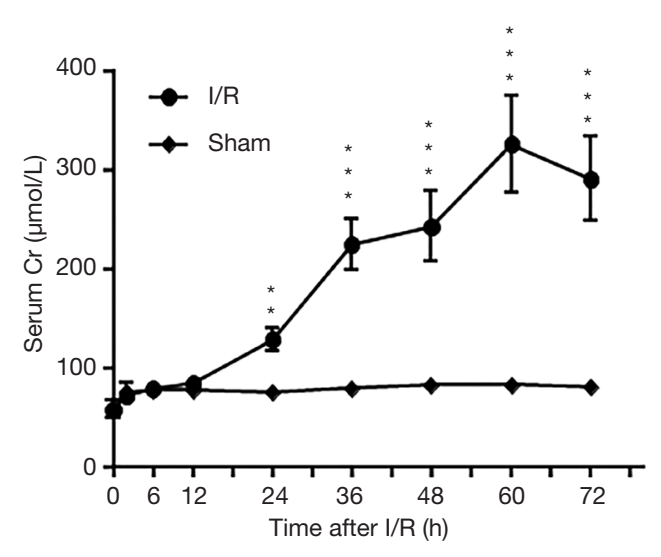

Figure 4 Detection of serum creatinine $(\mathrm{sCr})$ concentrations in sham and ischemic acute kidney injury (I-AKI) dogs. The serum samples were collected at the indicated times after ischemia/ reperfusion (I/R) and $\mathrm{sCr}$ was measured using VetTest $8008^{\circledR}$ Chemistry Analyzer and VT Analyzer Kit (IDEXX Laboratories, Lenexa, KS, USA). **, $\mathrm{P}<0.01$; *** $\mathrm{P}<0.001$.

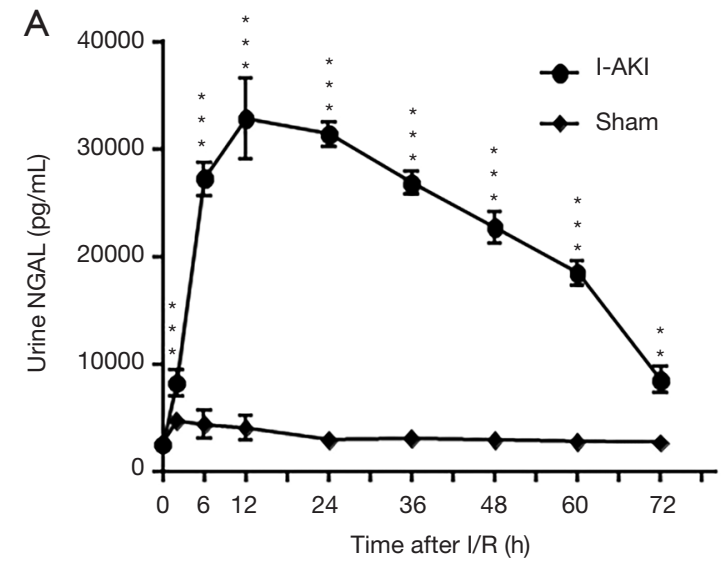

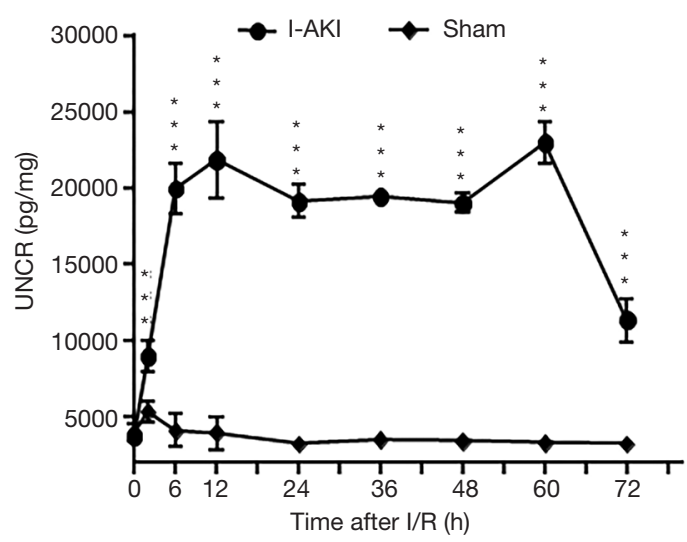

Figure 6 Detection of urinary neutrophil gelatinase-associated lipocalin creatinine ratio (uNCR) in sham and ischemic acute kidney injury (I-AKI) dogs. The urinary samples were collected at the indicated times after ischemia/reperfusion (I/R). Urinary neutrophil gelatinase-associated lipocalin (uNGAL) and creatinine were measured using VetTest $8008^{\circledR}$ Chemistry Analyzer and VT Analyzer Kit (IDEXX Laboratories, Lenexa, KS, USA) and a commercial NGAL enzyme-linked immunosorbent assay (ELISA) kit, respectively. ${ }^{* * *}, \mathrm{P}<0.001$.

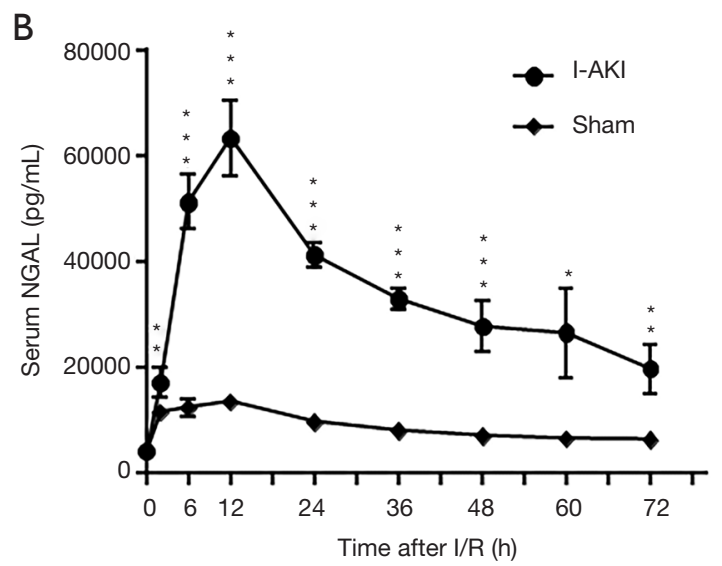

Figure 5 Detection of neutrophil gelatinase-associated lipocalin (NGAL) concentrations in sham and ischemic acute kidney injury (I-AKI) dogs. The urinary (A) and serum (B) samples were collected at the indicated times after ischemia/reperfusion (I/R), and NGAL concentrations were measured using a commercial NGAL enzyme-linked immunosorbent assay (ELISA) kit. ${ }^{*}, \mathrm{P}<0.05 ;{ }^{* *}, \mathrm{P}<0.01 ;{ }^{* * *}$, $\mathrm{P}<0.001$.

level from $6 \mathrm{~h}$ after I/R (Figure 6). In contrast, the average uNCR value of I-AKI dogs quickly rose from $2 \mathrm{~h}$, maintained at variably high levels $(20,008.37 \pm 1,641.02$ to $23,009.82 \pm 1,359 / 08 \mathrm{pg} / \mathrm{mg}$ ) from 6 to $60 \mathrm{~h}$, and then plummeted $(11,333.93 \pm 1,463.53 \mathrm{pg} / \mathrm{mg})$ by $72 \mathrm{~h}$ after I/R (Figure 6).

\section{ROC analysis of inclusion variables}

The ROC analysis showed that, among the 5 variables tested, uNCR had the highest area under the curve (AUC) value, followed by uNGAL, sNGAL, BUN, and $\mathrm{sCr}$ (Figure 7, Table 2). Similarly, both uNCR and uNGAL 


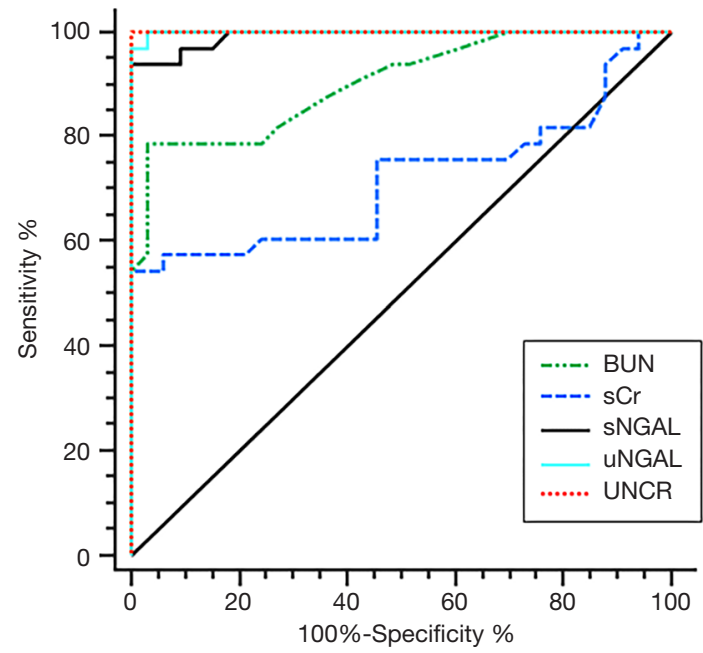

Figure 7 Comparative detection of sensitivities and specificities of different diagnostic criteria. The data were analyzed using the receiver operating characteristic (ROC) approach.

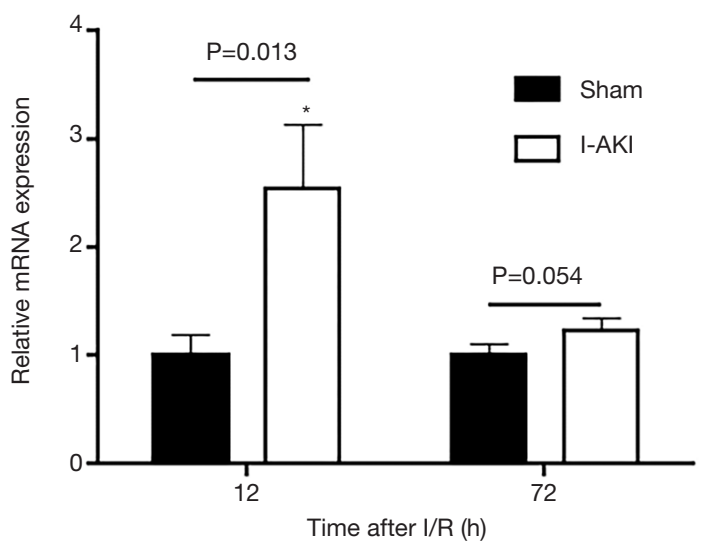

Figure 8 Detection of neutrophil gelatinase-associated lipocalin (NGAL) transcription in sham and ischemic acute kidney injury (I-AKI) dog kidneys. Kidney samples were collected at the indicated times and NGAL transcription was detected by quantitative real-time reverse transcription-polymerase chain reaction (qRT-PCR). *, $\mathrm{P}<0.05$.

Table 2 ROC analysis of the inclusion variables in this study

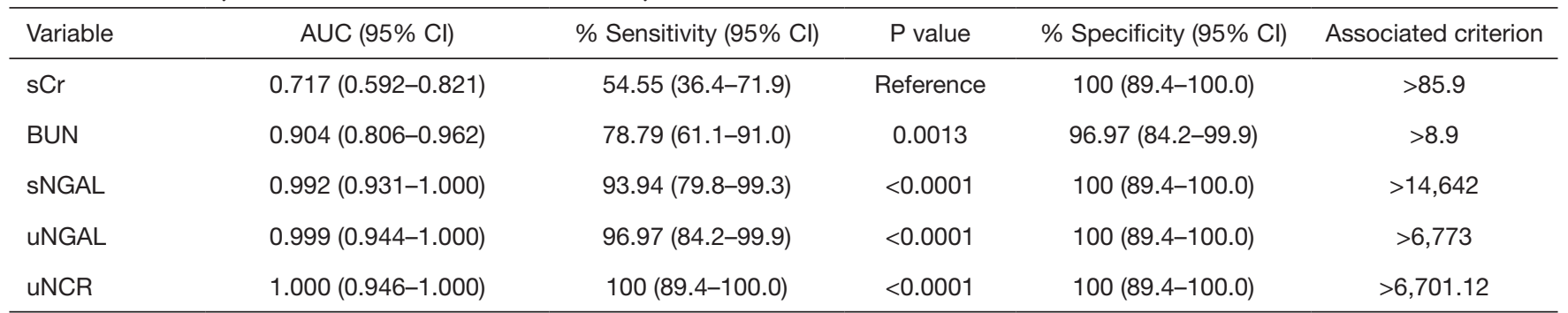

$\mathrm{ROC}$, receiver operating characteristic; AUC, area under the ROC curve; Cl, confidence interval; sCr, serum creatinine; BUN, blood urea nitrogen; sNGAL, serum NGAL; uNGAL, urinary NGAL; uNCR, uNGAL/uCr ratio.

had the highest (96.97 and 100\%, respectively) detection sensitivity, followed by sNGAL (93.94\%), BUN (78.79\%), and $\mathrm{s} \mathrm{Cr}(54.55 \%)$ (Figure 7, Table 2).

\section{Detection of NGAL expression}

Quantitative RT-PCR (qRT-PCR) analysis showed that, compared to that in the sham dog kidneys, NGAL transcription in the I-AKI dog kidneys increased by 2.7 folds at $12 \mathrm{~h}$ after I/R. By $72 \mathrm{~h}$ after I/R, NGAL transcription in the I-AKI dog kidneys decreased significantly, which was 1.2-fold higher than that in the sham dog kidneys (Figure 8). The immunohistochemical (IHC) analysis confirmed the NGAL expression patterns in the sham and I-AKI dog kidneys (Figure 9).

\section{Discussion}

Animal models of AKI have provided important insight into its underlying pathophysiology. Although rodent models of AKI have provided a great deal of information regarding pathophysiological mechanisms of injury and repair, they do not capture the complex comorbidities of heterogeneous patient populations (27). As large animal models for I/R injury, the dog is a good choice due to its similar behavior to that of human beings in regard to this function (28). In the present study, we established a canine model of I-AKI by renal I/R. Histopathological analysis showed that the 

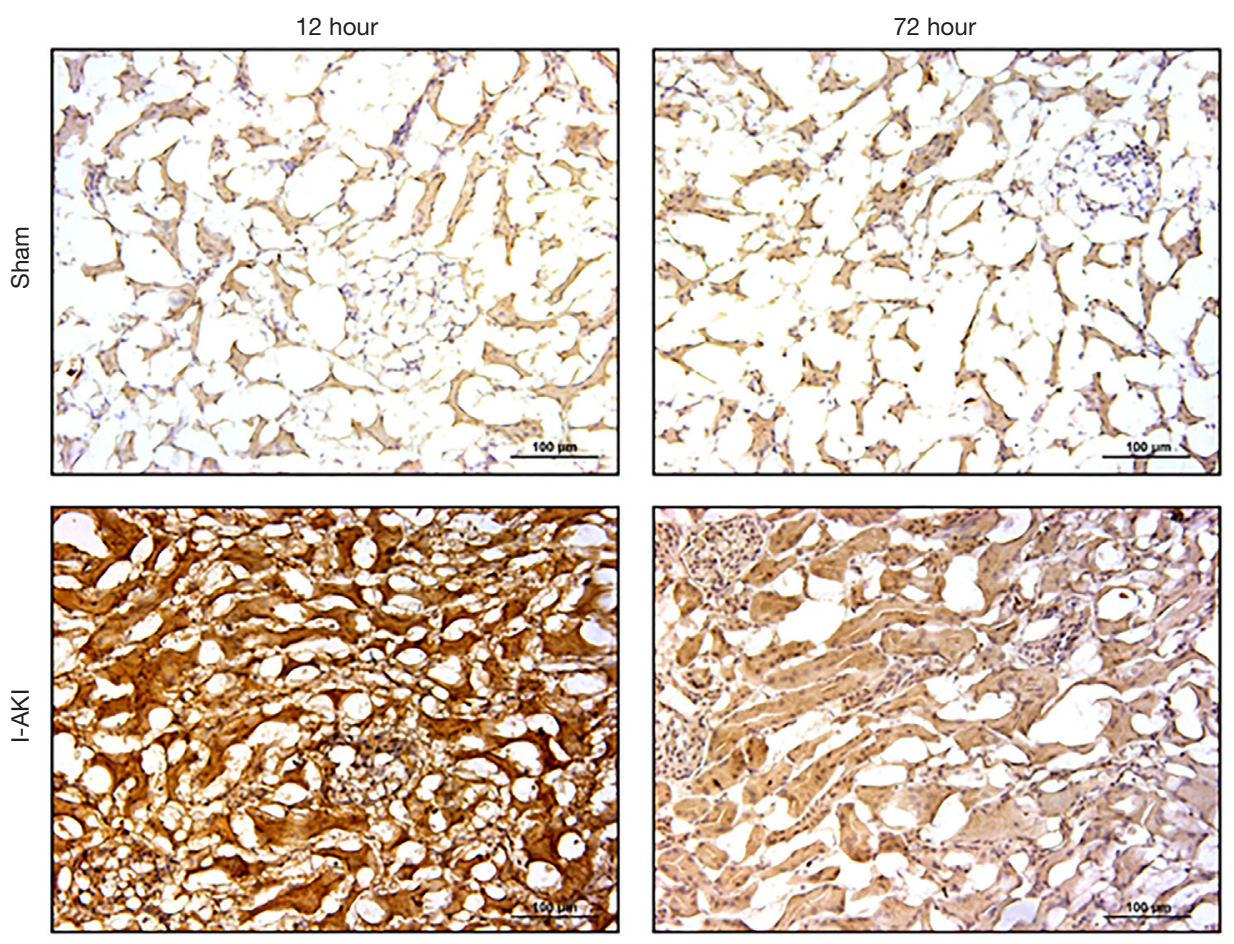

Figure 9 Detection of neutrophil gelatinase-associated lipocalin (NGAL) expression in sham and ischemic acute kidney injury (I-AKI) dog kidneys. Kidney samples were collected at the indicated times and NGAL expression was detected by immunobiochemical staining.

canine model had typical AKI lesions including necrosis of renal tubular epithelium and atrophy of renal tubules, at 72 $\mathrm{h}$ after I/R in particular. According to AKIN (Acute Kidney Injury Network) classification, the severity of the canine I/R-AKI could be classified as stage III (failure) since the increase in sCr was 3 times higher the normal level (7).

Novel early markers are required for the prevention, diagnosis, treatment, and prognosis prediction of AKI. Although several biomarkers have been described for renal ischemia or nephrotoxicity, most studies on AKI are still using $\mathrm{sCr}$ as the indicator of kidney injury despite its poor sensitivity, specificity, and accuracy. In addition, the increase of $\mathrm{sCr}$ was not evident until renal function is irreversibly damaged (7). In this study, the increase of sCr was not evident until $24 \mathrm{~h}$ after I/R. In summation, it could be concluded that $\mathrm{s} C r$ was inadequate for early diagnosis of canine I-AKI. Like sCr, BUN could not function as an early biomarker for canine I-AKI due to its delayed rising after I/R.

Recent evidence demonstrates that NGAL is closely associated with AKI. Several experimental and clinical studies have shown that the expression of uNGAL and
sNGAL increases significantly in AKI $(29,30)$. In particular, the uNGAL level is closely associated with the severity of kidney injury, and can be detected earlier than other AKI markers (31). Therefore, NGAL has the potential to act as an effective early biomarker of AKI. However, the utility of NGAL in early diagnosis of canine I-AKI has not yet been confirmed. To this end, our study measured uNGAL and sNGAL in I-AKI dogs at different time points following I/R. The results showed that both uNGAL and sNGAL rose to significantly high levels at $2 \mathrm{~h}$ after I/R, which was $20 \mathrm{~h}$ earlier than that of $\mathrm{s} C r$ or BUN. These data suggest that NGAL could be used for early diagnosis of canine I-AKI. Unlike sCr or BUN, which remained at higher levels from $24 \mathrm{~h}$ after I/R, both uNGAL and sNGAL rose to the peak level at $12 \mathrm{~h}$ and then decreased rapidly. The dynamic expression of NGAL in I-AKI dog kidneys was confirmed by qRT-PCR and IHC analyses.

Although we clarified that NGAL could be used as an early biological marker for ischemia-induced AKI in beagle dogs, further support is required from the statistics of clinical data which address the variations existing between individuals, such as breed, age, feeding, and healthy status, 
among others.

\section{Conclusions}

In conclusion, sNGAL, uNGAL, and uNCR are reliable biomarkers for the early diagnosis of AKI in the ischemiareperfusion AKI model, and in particular, UNCR is a more stable and durable biomarker.

\section{Acknowledgments}

Funding: This work was supported by the Graduate Research and Innovation Projects of Jiangsu Province (KYLX_1360) and the Priority Academic Program Development of Jiangsu Higher Education Institutions (PAPD). The sponsors did not have any influence on the study design, the collection, analysis, and interpretation of the data, writing of the manuscript, or on the decision to submission for publication.

\section{Footnote}

Reporting Checklist: The authors have completed the ARRIVE reporting checklist. Available at http://dx.doi. org/10.21037/atm-20-6314

Data Sharing Statement: Available at http://dx.doi. org/10.21037/atm-20-6314

Conflicts of Interest: All authors have completed the ICMJE uniform disclosure form (available at http://dx.doi. org/10.21037/atm-20-6314). The authors have no conflicts of interest to declare.

Ethical Statement: The authors are accountable for all aspects of the work in ensuring that questions related to the accuracy or integrity of any part of the work are appropriately investigated and resolved. All animal tests were approved by the Experimental Animal Committee of the Nantong University (20190307-012) and were conducted in accordance with the Guidelines for Experimental Animals of the Ministry of Science and Technology (Beijing, China).

Open Access Statement: This is an Open Access article distributed in accordance with the Creative Commons Attribution-NonCommercial-NoDerivs 4.0 International License (CC BY-NC-ND 4.0), which permits the non- commercial replication and distribution of the article with the strict proviso that no changes or edits are made and the original work is properly cited (including links to both the formal publication through the relevant DOI and the license). See: https://creativecommons.org/licenses/by-nc-nd/4.0/.

\section{References}

1. Mugford A, Li R, Humm K. Acute kidney injury in dogs and cats 1. Pathogenesis and diagnosis. In Practice 2013;35:253-64.

2. Amin AA, Alabsawy EI, Jalan R, et al. Epidemiology, Pathophysiology, and Management of Hepatorenal Syndrome. Semin Nephrol 2019;39:17-30.

3. Quenot JP, Dargent A, Large A, et al. Treatment of sepsisinduced acute kidney injury in the ICU: the therapeutic targets do not seem to be established yet. Ann Transl Med 2019;7:S181.

4. Li R, Mugford A, Humm K. Acute kidney injury in dogs and cats 2. Management, treatment and outcome. In Practice 2013;35:302-16.

5. Crane NJ, Huffman SW, Alemozaffar M, et al. Evidence of a heterogeneous tissue oxygenation: renal ischemia/ reperfusion injury in a large animal model. J Biomed Opt 2013;18:035001.

6. Thoen ME, Kerl ME. Characterization of acute kidney injury in hospitalized dogs and evaluation of a veterinary acute kidney injury staging system. J Vet Emerg Crit Care (San Antonio) 2011;21:648-57.

7. Lee YJ, Chang CC, Chan JPW, et al. Prognosis of acute kidney injury in dogs using RIFLE (Risk, Injury, Failure, Loss and End-stage renal failure)-like criteria. Vet Rec 2011;168:264.

8. Lin X, Yuan J, Zhao Y, et al. Urine interleukin-18 in prediction of acute kidney injury: a systemic review and meta-analysis. J Nephrol 2015;28:7-16.

9. Singh R, Dodkins J, Doyle J, et al. Acute Kidney Injury Biomarkers: What Do They Tell Us? Contrib Nephrol 2018;193:21-34.

10. Bonventre J. Kidney injury molecule-1 (KIM-1): a urinary biomarker and much more. Nephrol Dial Transplant 2009;24:3265-8.

11. Almy FS, Christopher MM, King DP, et al. Evaluation of cystatin $\mathrm{C}$ as an endogenous marker of glomerular filtration rate in dogs. J Vet Intern Med 2002;16:45-51.

12. Andreucci M, Faga T, Pisani A, et al. The ischemic/ nephrotoxic acute kidney injury and the use of renal biomarkers in clinical practice. Eur J Intern Med 


\section{Page 10 of 10}

2017;39:1-8.

13. Srisawat N, Kellum JA. The Role of Biomarkers in Acute Kidney Injury. Crit Care Clin 2020;36:125-40.

14. Supavekin S, Zhang W, Kucherlapati R, et al. Differential gene expression following early renal ischemia/reperfusion. Kidney Int 2003;63:1714-24.

15. Wagener G, Jan M, Kim M, et al. Association between increases in urinary neutrophil gelatinase-associated lipocalin and acute renal dysfunction after adult cardiac surgery. Anesthesiology 2006;105:485-91.

16. Kjeldsen L, Cowland JB, Borregaard N. Human neutrophil gelatinase-associated lipocalin and homologous proteins in rat and mouse. Biochim Biophys Acta 2000;1482:272-83.

17. Lee YJ, Hu YY, Lin YS, et al. Urine neutrophil gelatinaseassociated lipocalin (NGAL) as a biomarker for acute canine kidney injury. BMC Vet Res 2012;8:248.

18. Koyner JL, Garg AX, Coca SG, et al. Biomarkers predict progression of acute kidney injury after cardiac surgery. J Am Soc Nephrol 2012;23:905-14.

19. Budsberg SC, Torres BT, Kleine SA, et al. Lack of effectiveness of tramadol hydrochloride for the treatment of pain and joint dysfunction in dogs with chronic osteoarthritis. J Am Vet Med Assoc 2018;252:427-32.

20. Amdisen C, Keller AK, Hansen RS, et al. Testing Danegaptide Effects on Kidney Function after Ischemia/ Reperfusion Injury in a New Porcine Two Week Model. PLoS One 2016;11:e0164109.

21. Lee G, Jeon S, Lee S, et al. Quantitative evaluation of renal parenchymal perfusion using contrast-enhanced ultrasonography in renal ischemia-reperfusion injury in dogs. J Vet Sci 2017;18:507-14.

22. Jiang B, Liu X, Chen H, et al. Ischemic postconditioning attenuates renal ischemic/reperfusion injury in mongrel dogs. Urology 2010;76:1519.e1-7.

Cite this article as: Cao J, Lu X, Gao F, Zhang X, Xia X, Sun H. Assessment of neutrophil gelatinase-associated lipocalin as an early biomarker for canine renal ischemia-reperfusion injury. Ann Transl Med 2020;8(22):1491. doi: 10.21037/atm-206314

\section{Cao et al. NGAL is canine ischemic AKI early diagnostic biomarker}

23. Karim N, Rahman A, Chanudom L, et al. Mangosteen Vinegar Rind from Garcinia mangostana Prevents HighFat Diet and Streptozotocin-Induced Type II Diabetes Nephropathy and Apoptosis. J Food Sci 2019;84:1208-15.

24. Zhou X, Ma B, Lin Z, et al. Evaluation of the usefulness of novel biomarkers for drug-induced acute kidney injury in beagle dogs. Toxicol Appl Pharmacol 2014;280:30-5.

25. Wong A, Cianciolo RE. Comparison of immunohistochemistry and immunofluorescence techniques using anti-lambda light chain antibodies for identification of immune complex deposits in canine renal biopsies. J Vet Diagn Invest 2018;30:721-7.

26. Matsuura R, Komaru Y, Miyamoto Y, et al. Response to different furosemide doses predicts AKI progression in ICU patients with elevated plasma NGAL levels. Ann Intensive Care 2018.

27. Zuk A, Bonventre JV. Acute Kidney Injury. In: Caskey CT, editor. Annual Review of Medicine, Vol 67. Annu Rev Med 2016:293-307.

28. Modolo NS, Castiglia YM, Ganem EM, et al. Acute renal ischemia model in dogs: effects of metoprolol. Ren Fail 2001;23:1-10.

29. Monari E, Troia R, Magna L, et al. Urine neutrophil gelatinase-associated lipocalin to diagnose and characterize acute kidney injury in dogs. J Vet Intern Med 2020;34:176-85.

30. Cortellini S, Pelligand L, Syme H, et al. Neutrophil Gelatinase-Associated Lipocalin in Dogs With Sepsis Undergoing Emergency Laparotomy: A Prospective CaseControl Study. J Vet Intern Med 2015;29:1595-602.

31. Shang $W$, Wang $Z$. The Update of NGAL in Acute Kidney Injury. Curr Protein Pept Sci 2017;18:1211-7.

(English Language Editor: J. Jones) 